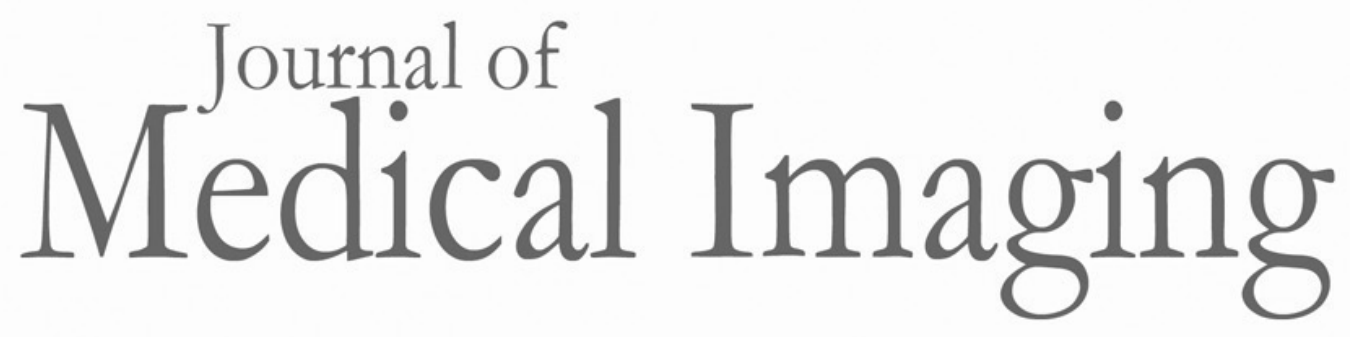

Medicallmaging.SPIEDigitalLibrary.org

\title{
Special Section Guest Editorial: Medical Image Perception and Observer Performance
}

\author{
William F. Auffermann \\ Trafton Drew \\ Elizabeth A. Krupinski
}




\title{
Special Section Guest Editorial: Medical Image Perception and Observer Performance
}

\author{
William F. Auffermann, ${ }^{\text {a,* }}$ Trafton Drew, ${ }^{\mathrm{b}}$ and \\ Elizabeth A. Krupinskic \\ ${ }^{a}$ University of Utah School of Medicine, Department of Radiology and Imaging Sciences, \\ Salt Lake City, Utah, United States \\ ${ }^{b}$ University of Utah, Department of Psychology, Salt Lake City, Utah, United States \\ ${ }^{c}$ Emory University, Department of Radiology and Imaging Sciences, Atlanta, Georgia, \\ United States
}

The interdisciplinary field of medical image perception endeavors to better understand the role of perceptual and cognitive factors in medical image interpretation. The Medical Image Perception Society (MIPS) holds a biannual conference where radiologists, physicists, engineers, statisticians, psychologists, and others meet to discuss the latest research on medical image perception. The MIPS XVIII Conference was held July 14 through July 17, 2019, at the University of Utah, Salt Lake City, Utah, USA. Our next MIPS meeting will be held in 2021 in York, United Kingdom.

The MIPS meeting is not only a venue for presenting and discussing the latest medical image perception research, but also sponsors trainees' attendance and participation. The MIPS XVIII meeting provided financial support for trainee attendance using NIBIB/NCI R13EB028683-01 and through MIPS member dues. There were eight MIPS trainee scholars from seven institutions and four countries. All scholars had abstracts that were accepted and presented at the conference. MIPS Scholars included: Seungyeon Choi (South Korea), Lisa Heisterberg (Australia), Ziba Gandomkar (Australia), Aarthi Ganesan (Australia), Nada Kamona (United States), Lucie Lévêque (China), Hayden Schill (United States), Melissa Treviño (United States).

The keynote presentation titled "A clinician's perspective on diagnostic accuracy," was given by Joann Elmore, MD, David Geffen School of Medicine University of California Los Angeles, United States. Dr. Elmore's talk examined the under-recognized perils of suboptimal inter-reader variability for interpretation of pathology specimens. The conference was organized into themebased sessions of short presentations, with a total of 38 talks presented. Speakers and attendees were invited to submit articles for this special section of Volume 7, Issue 2 of the Journal of Medical Imaging, ultimately resulting in the 11 peer-reviewed, perception-focused papers discussed below.

Radiology Work Environment: Dabrowiecki et al. examined the effects of blue light filtering glasses on computer vision syndrome and fatigue in radiology residents. The results of their study show a majority of symptoms of computer vision symptoms (CVS) decreased, although not to a statistically significant degree. They also noted some interesting gender-related effects, as well as those related to year of residency. It appears that senior residents are already becoming better than junior residents at dealing with fatigue and CVS, although more research is needed.

Breast Cancer Detection: Breast cancer research is always an important topic at MIPS meetings and 2019 had some very interesting presentations on this topic, with three being published in this special section. Wu et al. examined the role of gist/gestalt processing of digital breast tomosynthesis (DBT) images. Their study suggests that gist processing plays a significant role in DBT image evaluation, and not surprisingly the more experienced the observer the better their ability to detect lesions in a $1.5 \mathrm{sec}$ glance at the image. Schwartz et al. evaluated the interpretation time for screening mammography with respect to the number of CAD (computer-aided detection) markings and found that the interpretation time increased proportional to the number of markings, and that markings were reassuringly not disregarded even when their number was large. Lago et al. examined the useful field of view (UFOV) for differing imaging modalities

\footnotetext{
*Address all correspondence to William F. Auffermann, E-mail: WILLIAM.AUFFERMANN@hsc.utah.edu

(C) 2020 Society of Photo-Optical Instrumentation Engineers (SPIE)
} 
(single slices CT, 2-D mammograms, single slices DBT) and types of targets, finding that the useful field of view varies as related to these and other variables. For example, the UVOF for liver lesions was found to be over twice as large as that for calcifications in mammograms and DBT images.

CAD/Deep Learning: Leung et al. examined using fully automated quantitative CT evaluation for emphysema. The presented method performed well relative to conventional methods for emphysema assessment. Such automated quantitative methods may prove useful for radiologists who are already overburdened during their clinical workday. Du-Crow et al. explored whether the availability of CAD changes a reader's performance when evaluating for suspicious microcalcifications on breast images. Their results suggest that the availability of CAD alters the initial search process, illustrating some of the challenges of implementing $\mathrm{CAD}$ in a clinical environment. In his pilot study, Hegdé used deep learning algorithms to teach naive observers to detect signs of cancer on breast imaging studies. He found that not only did the subjects learn to perform that task better with training, but their visual search patterns appear to have changed as well.

Gist/Gestalt Processing: Treviño et al. studied perceptual processing of 2D and 3D prostate images and found that gist image processing may play a role in evaluation complex 3D medical images. This study nicely parallels the Wu et al. study as it also found that experienced readers are especially able to extract data from complex radiographic images in very brief presentations (as short as $48 \mathrm{msec}$ in some cases).

Modeling Performance: Banerjee et al. examined whether the improved performance seen after training observers in a search pattern results from learning a new perceptual skill or is merely the result of a shift of attention to the task at hand. Results of their study suggest perceptual learning occurs, and supports the notion that perceptual training may be a useful adjunct to conventional educational methods in radiology. Li et al. examined the influence of background lung characteristics on nodule detection using CT and found that locally distracting features decreased the detectability of lung nodules, replicating and extending prior work that found similar effects with plain film chest x-ray images. ${ }^{1}$ Gabrani-Juma et al. developed a method for simulation of lesions in positron emission tomography (PET) studies, and examined lesion detectability as a function of lesion and size contrast.

The papers in this special section reflect the breadth and depth of topics that fall under the medical image perception umbrella. It was interesting to see at MIPS XVIII how certain topics (e.g., gist) continue to be of interest to researchers (many of the first studies on this topic were conducted in radiology back in the 1980s) and how new technologies (e.g., machine and deep learning) draw the attention of this important group of researchers. Hopefully those not in the field of medical image perception will learn from these studies and start to incorporate some of these findings into their own work. For example, the work on gist, search patterns, and the ways readers interact with $\mathrm{CAD}$ all have potential to be used in the development of new types of educational and training tools, especially in this age of artificial intelligence. We hope these papers are of interest, and invite readers to join us at our next MIPS XIX meeting in 2021 in York, England.

\section{References}

1. T. B. Smith et al., "Local complexity metrics to quantify the effect of anatomical noise on detectability of lung nodules in chest CT imaging," J. Med. Imaging 5(4), 045502 (2018).

William F. Auffermann is a cardiothoracic radiologist and associate professor of radiology and imaging sciences at the University of Utah. He received his $\mathrm{MD} / \mathrm{PhD}$ from the University of Minnesota in 2004. His research interests include medical image perception, medical education, and the application of perception research to new educational methods using simulation.

Trafton Drew is an assistant professor in the Department of Psychology at the University of Utah. He received his $\mathrm{PhD}$ from the University of Oregon in 2009. His research interests focus on understanding the real-world ramifications and underlying neural mechanisms of visual attention and studying radiologist behavior to understand why errors are sometimes made and ways to potentially reduce the number of errors committed. 
Elizabeth A. Krupinski is a professor and vice-chair for Research at Emory University in the Department of Radiology and Imaging Sciences and a subject matter expert for the Emory Telehealth Program. She received her BA degree from Cornell, MA degree from Montclair State, and $\mathrm{PhD}$ from Temple, all in experimental psychology. Her interests are in medical image perception, observer performance, decision making, and human factors. She is an associate director of evaluation for the Arizona Telemedicine Program and co-director of the Southwest Telehealth Resource Center. She is a former president of ATA, former chair of Society for Imaging Informatics in Medicine, former chair of the SPIE Medical Imaging Conference, and president of the Medical Image Perception Society. She is co-editor of the Journal of Telemedicine and Telecare and on the editorial board of the journal Telemedicine and E-Health. 